\title{
iStent inject Trabecular Micro-Bypass with or Without Cataract Surgery Yields Sustained 5-Year Glaucoma Control
}

\author{
Fritz H. Hengerer - Gerd U. Auffarth · Ina Conrad-Hengerer
}

Received: November 7, 2021 / Accepted: December 23, 2021 / Published online: February 3, 2022

(c) The Author(s) 2022

\section{ABSTRACT}

Introduction: This study evaluated the 5-year effectiveness and safety of iStent inject ${ }^{\circledR}$ trabecular micro-bypass with or without cataract surgery (Combined or Standalone, respectively) in patients with open-angle glaucoma (OAG).

Methods: This prospective longitudinal case series included consecutive iStent inject cases from a single surgeon at a large German academic hospital. Intraocular pressure (IOP), medications, safety, and indicators of disease stability through 5 years were assessed in the Overall cohort and in subgroup analyses stratified by usage (Combined or Standalone).

Results: Preoperative mean IOP in the Overall cohort $(n=125)$ was $23.5 \pm 6.2 \mathrm{mmHg}$ on $2.68 \pm 1.02$ mean medications, reducing to $14.1 \pm 1.8 \mathrm{mmHg}$ on $0.77 \pm 0.82$ medications at 5 years ( $40 \%$ and $71 \%$ reductions, respectively; both $p<0.001)$. All but 1 eye $(>99 \%)$ were on

F. H. Hengerer

Department of Ophthalmology, Buergerhospital, Frankfurt, Germany

F. H. Hengerer - G. U. Auffarth .

I. Conrad-Hengerer $(\bowtie)$

International Vision Correction Research Centre

(IVCRC), David J Apple International Laboratory of

Ophthalmic Pathology, Department of

Ophthalmology, University Eye Hospital, University

of Heidelberg, Im Neuenheimer Feld 400,

69120 Heidelberg, Germany

e-mail: mail@augenarzt-trebur.de medication(s) preoperatively, but $46 \%$ were medication-free at 5 years $(p<0.001)$. In Combined eyes $(n=81)$, mean IOP decreased by $39 \%$ (22.6 $\mathrm{mmHg}$ to $13.8 \mathrm{mmHg}, p<0.001$ ) and medications by $69 \%$ ( 2.52 to $0.78, p<0.001)$. In Standalone eyes, mean IOP reduced by $42 \%$ (25.3 $\mathrm{mmHg}$ to $14.6 \mathrm{mmHg}, p<0.001$ ) and medications by $75 \%$ (2.98 to $0.74, p<0.001)$. At final follow-up, $83 \%$ of eyes had achieved $\geq 20 \%$ IOP reduction, and all but 1 eye (>99\%) had the same or lower IOP versus preoperative; all eyes (100\%) maintained or reduced their medication burden versus preoperative. Favorable safety included 0 intraoperative complications and 0 filtration surgeries through 5 years. Long-term indicators of disease stability (visual fields, retinal nerve-fiber layer thickness, and cup:disc ratio) were unchanged over the course of 5-year follow-up.

Conclusions: iStent inject produced significant and durable 5-year reductions in IOP (nearly $10-\mathrm{mmHg}$ reduction) and medications (nearly 2-medication reduction), with stable disease parameters over time. Combined and Standalone subgroups had similar outcomes.

Keywords: Microinvasive glaucoma surgery (MIGS); iStent inject; Trabecular bypass; Cataract; Long-term; Intraocular pressure; Second-generation; Stent 


\section{Key Summary Points}

Why carry out this study?

Safe and efficacious long-term surgical treatments are needed to prevent or minimize vision loss from glaucoma, a major cause of global blindness.

This prospective study assessed real-world 5 -year outcomes following implantation of two second-generation trabecular micro-bypass stents either with or without concomitant cataract surgery in eyes with various severities of open-angle glaucoma.

The study hypothesized that this treatment modality could effectively and safely reduce intraocular pressure (IOP) and medication burden over the long term in these glaucomatous eyes, while maintaining stability of long-term disease measures.

\section{What was learned from the study?}

The data demonstrated substantial 5-year reductions in IOP and medications, along with favorable safety, in eyes with a considerable preoperative medication burden and frequent history of glaucoma surgery. Long-term indicators of disease stability (visual fields, retinal nerve-fiber layer thickness, and cup:disc ratio) were unchanged over the course of 5-year follow-up. Results were similarly favorable in combined and standalone usage.

Drawing upon long-term, clinically heterogeneous, real-world patient data, this study provides directly relevant information for clinicians and patients evaluating glaucoma treatment options.

\section{INTRODUCTION}

The development of micro-invasive glaucoma surgery (MIGS) has fundamentally altered the way glaucoma is treated worldwide. Constituting virtually no procedures in the United States (US) in 2011, these surgeries became the most commonly-performed incisional glaucoma procedures by 2018 and are expected to further increase over time [1]. Among the various MIGS options, the iStent trabecular micro-bypass (the first US Food and Drug Administration-approved device, in 2012)] and the second-generation iStent inject trabecular micro-bypass (containing 2 stents, each with modified design vs. the original) have accumulated the most diverse and long-term MIGS evidence base yet available [2]. The stents have been studied in various races/ethnicities, glaucoma types, severities, and usages (combined or standalone). Studies and subsequent publications have been completed in at least 20 countries, and to date there are at least 15 publications with 4-year or longer follow-up data [2]. Regarding iStent inject specifically, Germany was one of several countries around the world to rapidly adopt the technology, thereby allowing for some of the largest and longest-term datasets yet available on iStent inject effectiveness and safety.

Prospective 3-year findings from the present cohort have been previously published for both Combined and Standalone eyes [3, 4]. At 3 years postoperative, mean IOP and medication reductions in Combined eyes were $37 \%$ and $68 \%$, respectively, and were $42 \%$ and $88 \%$ in Standalone eyes, respectively. In the current paper, we report the 5-year outcomes for the entire cohort, with subgroup analysis of Combined and Standalone eyes.

This report provides some of the first 5-year real-world iStent inject data yet available in the literature. By including consecutive patients (i.e. all-comers) rather than pre-selecting a narrow slice of the population, the results are broadly applicable to surgeons' actual practices. The inclusion of subgroup stratification by combined-versus-standalone usage may further enhance generalizability and relevance. Subgroup analyses also allow us to discern the independent effect of the stents themselves, apart from those of cataract extraction. And, finally, the prospective longitudinal design and high accountability through 5 years help ensure the reliability of the findings. 
Table 1 Demographics and preoperative characteristics $(n=125)$

\begin{tabular}{|c|c|c|}
\hline Mean \pm SD (unless noted otherwise) & $\begin{array}{l}\text { Combined } \\
\text { iStent inject + Phaco } \\
\text { (81 eyes) }\end{array}$ & $\begin{array}{l}\text { Standalone } \\
\text { iStent } \text { inject } \\
\text { (44 eyes) }\end{array}$ \\
\hline Age (years) & $72.7 \pm 7.5$ & $71.3 \pm 10.5$ \\
\hline Range (years) & $54-86$ & $40-88$ \\
\hline Gender & $56 \%$ male $/ 44 \%$ female & $52 \%$ male $/ 48 \%$ female \\
\hline Cup:disc ratio & $0.74 \pm 0.17$ & $0.78 \pm 0.18$ \\
\hline VF MD & $-6.6 \pm 2.8 \mathrm{~dB}$ & $-7.0 \pm 3.5 \mathrm{~dB}$ \\
\hline Retinal nerve fiber layer thickness $(\mu \mathrm{m})$ & $81.4 \pm 12.3$ & $82.1 \pm 13.6$ \\
\hline Race/ethnicity & $100 \%$ non-Hispanic white & $100 \%$ non-Hispanic white \\
\hline Eyes with prior glaucoma procedures (\%) & $26(32 \%)$ & $22(50 \%)$ \\
\hline Prior glaucoma procedures & 31 procedures in 26 eyes & 35 procedures in 22 eyes \\
\hline Trabeculectomy & 12 & 13 \\
\hline $\mathrm{CPC}$ & 10 & 14 \\
\hline ALT/SLT & 5 & 3 \\
\hline Laser iridotomy & 2 & 2 \\
\hline Surgical iridectomy & 1 & 0 \\
\hline Single first-gen iStent & 0 & 1 \\
\hline XEN implantation & 1 & 2 \\
\hline Type of glaucoma & $n(\%)$ & $n(\%)$ \\
\hline POAG & $60(74 \%)$ & $38(86 \%)$ \\
\hline PXG & $15(19 \%)$ & $4(9 \%)$ \\
\hline Combined-Mechanism & $4(5 \%)$ & $1(2 \%)$ \\
\hline Pigmentary & $1(1 \%)$ & $0(0 \%)$ \\
\hline Secondary (neovascular/CRVO) & $1(1 \%)$ & $1(2 \%)$ \\
\hline Mean medicated IOP & $22.6 \pm 6.2 \mathrm{mmHg}$ & $25.3 \pm 6.0 \mathrm{mmHg}$ \\
\hline Mean \# medications & $2.52 \pm 1.06$ & $2.98 \pm 0.88$ \\
\hline Medicated IOP (mmHg) & $n(\%)$ & $n(\%)$ \\
\hline$\leq 15 \mathrm{mmHg}$ & $1(1 \%)$ & $1(2 \%)$ \\
\hline$\leq 18 \mathrm{mmHg}$ & $22(27 \%)$ & $4(9 \%)$ \\
\hline Medication burden & $n(\%)$ & $n(\%)$ \\
\hline Eyes med-free & $1(1 \%)$ & $0(0 \%)$ \\
\hline
\end{tabular}


Table 1 continued

\begin{tabular}{lll}
\hline Mean \pm SD (unless noted otherwise) & $\begin{array}{l}\text { CombinediStent } \\
\text { inject + Phaco(81 eyes) }\end{array}$ & $\begin{array}{l}\text { StandaloneiStent } \\
\text { inject(44 eyes) }\end{array}$ \\
\hline Eyes on 3-5 preop meds & $44(54 \%)$ & $33(75 \%)$ \\
\hline
\end{tabular}

$I O P$ intraocular pressure, $S D$ standard deviation, $C: D$ cup:disc, $V F M D$ visual field mean deviation, Preop preoperative, $P O A G$ primary open-angle glaucoma, $P X G$ pseudoexfoliative glaucoma, $C R V O$ central retinal vein occlusion, CPC cyclophotocoagulation, $A L T$ argon laser trabeculoplasty, $S L T$ selective laser trabeculoplasty; Med medication, Preop preoperative, Phaco phacoemulsification

\section{METHODS}

\section{Study Design and Participants}

In this prospective, longitudinal, single-surgeon study, all consecutive patients undergoing iStent inject implantation with or without cataract surgery were enrolled and followed for 5 years at an academic eye hospital in Germany. Patient recruitment occurred from March 2013 to December 2015; surgeries were completed from May 2013 to February 2016; and final data collection and analysis were completed by March 2021. To be enrolled in the study, patients were required to have a diagnosis of glaucoma [including primary open-angle glaucoma, pseudoexfoliative glaucoma (PXG), combined-mechanism glaucoma, pigmentary glaucoma, or secondary (neovascular) glaucoma]; have a history of inadequate response to prior surgical and medical glaucoma therapy; and to be in need of cataract surgery (for the Combined subgroup). Exclusion criteria consisted of corneal opacities preventing gonioscopic visualization, angle-closure glaucoma, pregnancy, congenital glaucoma, age under 18 years, and active ocular inflammation. Visits followed the surgeon's standard postoperative schedule which included, at minimum, a preoperative visit and postoperative visits at Day 1 , Week 1, Month 1, and Months 3, 6, 12, 24, 36, 48 , and 60 .

\section{Study Outcomes and Data Analysis}

Effectiveness measures included mean and percent reduction in IOP and medications versus preoperative. Proportional analyses for effectiveness included percent of eyes achieving the following outcomes: $\geq 20 \%$ IOP reduction versus preoperative; final IOP of $\leq 18 \mathrm{mmHg}$ and $\leq 15 \mathrm{mmHg}$; same or lower IOP versus preoperative; same or lower medication burden versus preoperative; and treatment with 0 medications or $\geq 3$ medications. Safety endpoints included intraoperative and postoperative adverse events, secondary surgical interventions, and proportion of eyes with traditional filtration surgery (trabeculectomy or tube shunt) over 5 years. In addition, long-term indicators of disease stability were measured and compared between the preoperative and 5-year visits; this included visual field mean deviation (VF MD), retinal nerve fiber layer thickness (RNFL), and cup:disc ratio (C:D ratio).

All IOP readings were measured by Goldmann applanation. Preoperative IOP was based on readings at two or more preoperative visits; postoperative IOP was based on three measurements per time point. The study was approved by the institutional review board of the University of Heidelberg, and complied with the Helsinki Declaration. All participants provided informed consent prior to enrollment.

Changes in IOP or medications from preoperative were assessed via paired $t$ test (for continuous variables) or Chi-square test (for categorical variables). Results were calculated and reported for the Overall cohort and for the Combined and Standalone subgroups. 

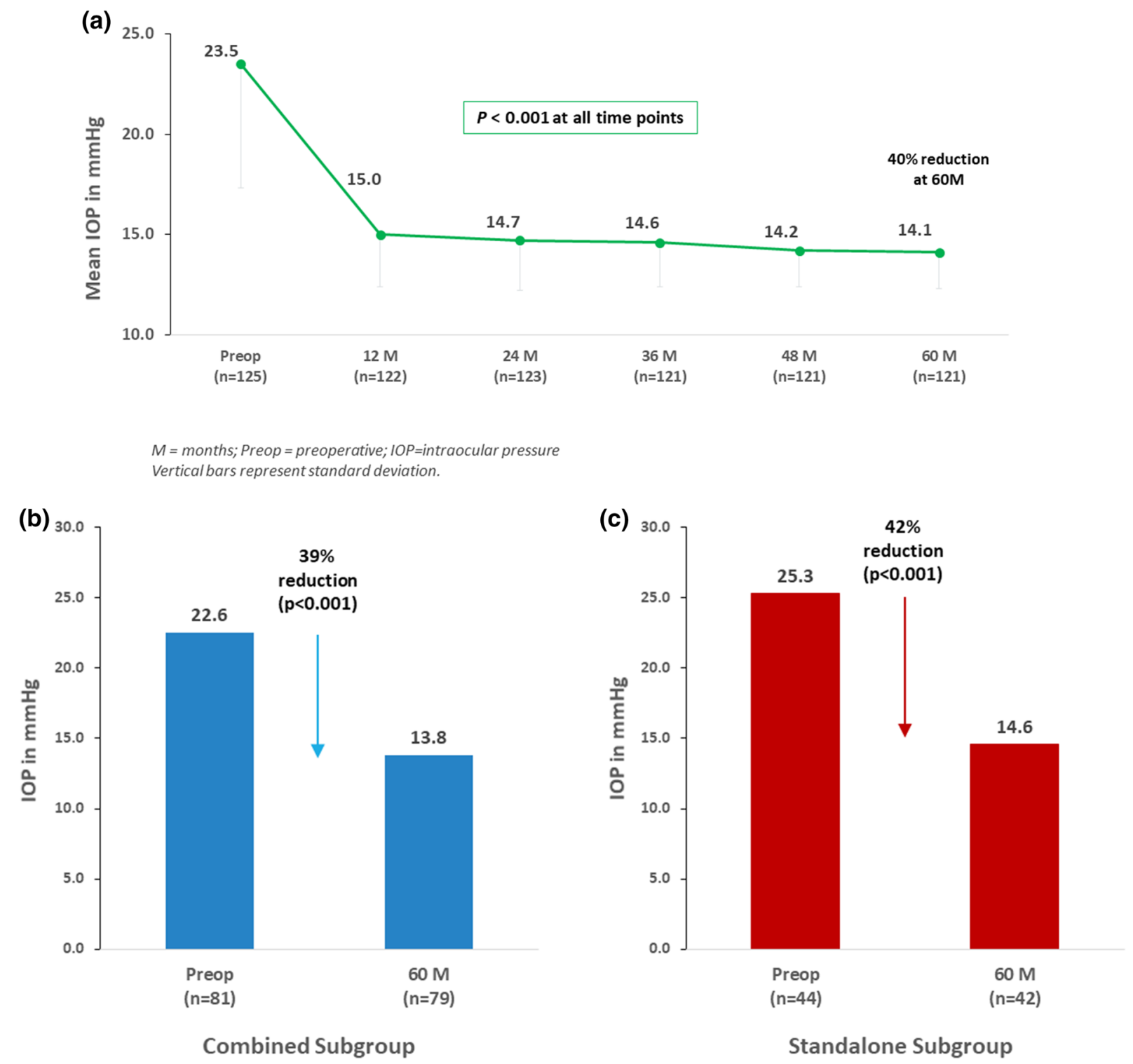

$M=$ months; Preop $=$ preoperative,$I O P=$ intraocular pressure .

Fig. 1 a Mean intraocular pressure (IOP) through 5 years postoperative in overall cohort. b (left) Mean IOP at 5 years versus preoperative: combined subgroup. c (right) Mean IOP at 5 years versus preoperative: standalone subgroup

\section{iStent inject Device and Surgical Implantation Technique}

The iStent inject device is comprised of two biocompatible titanium stents preloaded on a single-use sterile injector. Each stent contains four lateral outlet lumens to facilitate multidirectional outflow. The stents are placed $a b$ internally through the trabecular meshwork into Schlemm's Canal at approximately $2-3 \mathrm{~h}$ apart, providing access to up to $6 \mathrm{~h}$ of aqueous outflow [5, 6]. Implantation is completed either following phacoemulsification (in combinedcataract cases) or as a standalone procedure, as described in the respective prior publications $[3,4]$. Following implantation, stent position and patency are confirmed via gonioscopy, viscoelastic is removed, and surgical wound 


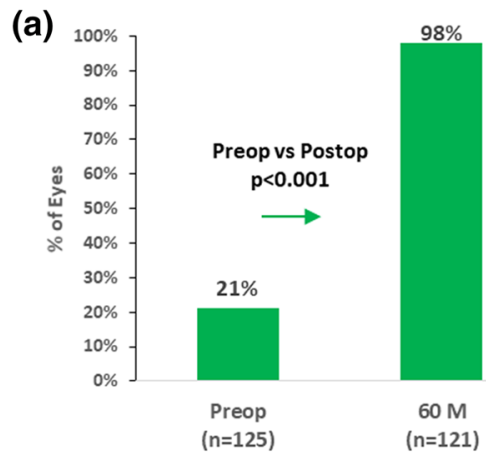

IOP $\leq 18 \mathrm{mmHg}$

$M=$ months; Preop = preoperative;

Posto $p=$ postoperative $I O P=$ intraocular pressure. (b)

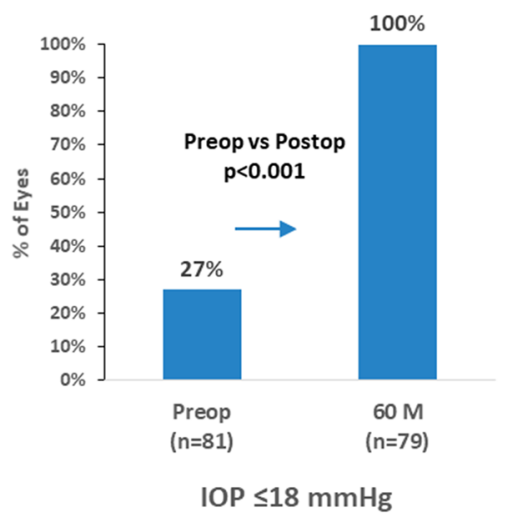

$M=$ months; Preop $=$ preoperative;

Postop=postoperative $1 O P=$ intraocular pressure.

(c)

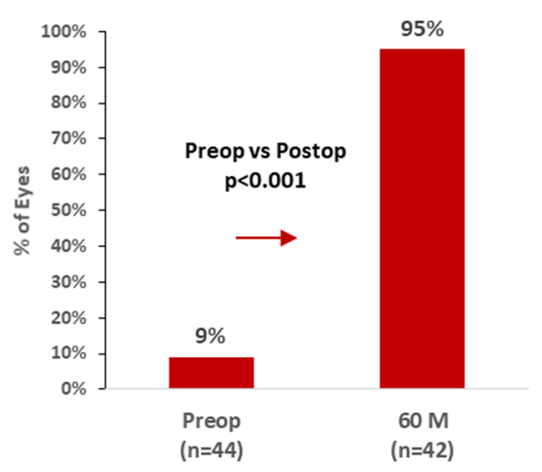

IOP $\leq 18 \mathrm{mmHg}$
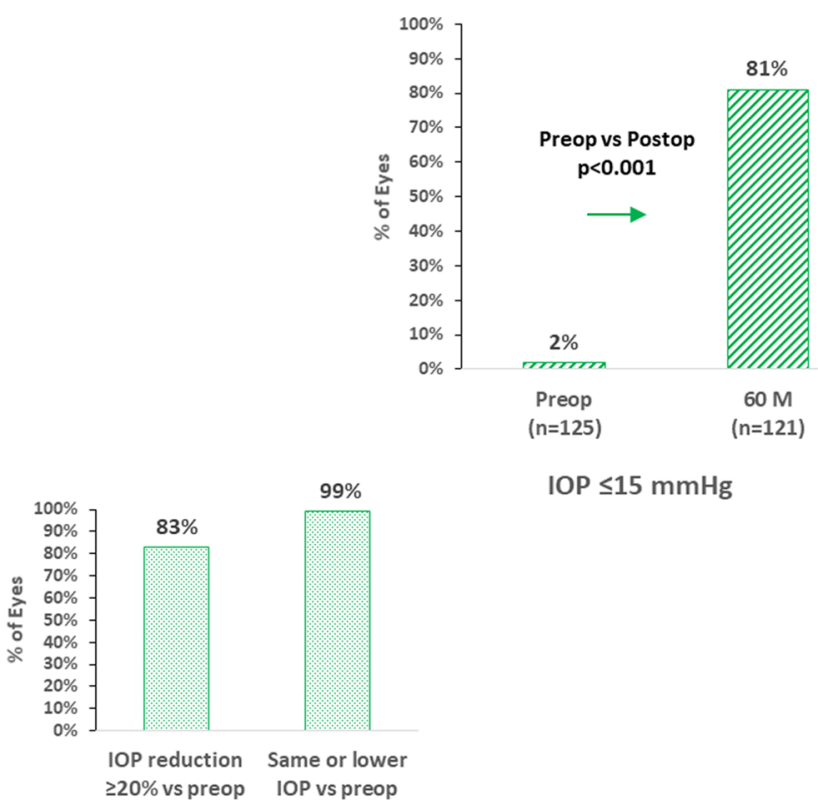

IOP $\leq 15 \mathrm{mmHg}$

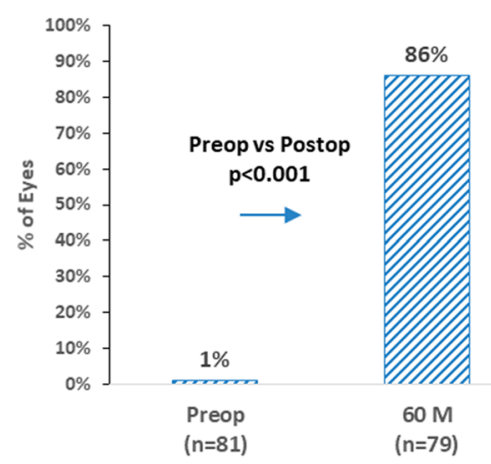

IOP $\leq 15 \mathrm{mmHg}$

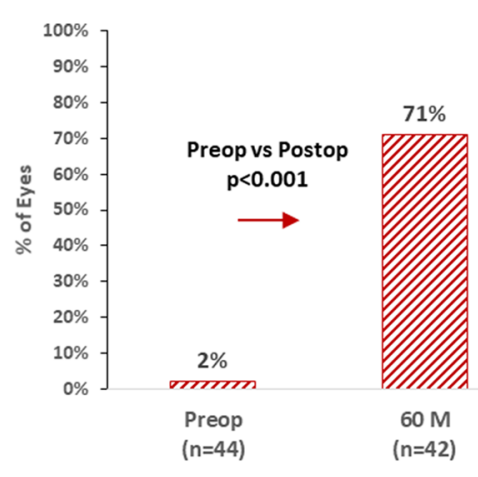

IOP $\leq 15 \mathrm{mmHg}$ 
4Fig. 2 a. Proportional analysis of IOP outcomes at 5 years versus preoperative in overall cohort: IOP $\leq 18 \mathrm{mmHg}$, IOP $\leq 15 \mathrm{mmHg}$, IOP reduction $\geq 20 \%$ versus preoperative, and eyes with same or lower IOP versus preoperative. b Proportional analysis of IOP Outcomes at 5 years versus preoperative in combined subgroup: IOP $\leq 18 \mathrm{mmHg}$, IOP $\leq 15 \mathrm{mmHg}$. c Proportional analysis of IOP outcomes at 5 years versus preoperative in standalone subgroup: IOP $\leq 18 \mathrm{mmHg}$, IOP $\leq 15 \mathrm{mmHg}$

closure is ensured. Postoperatively, patients were prescribed 1 week of topical antibiotic ciprofloxacin (Floxal ${ }^{\circledR}$, Bausch\&Lomb, Berlin, Germany) and four weeks of topical anti-inflammatory medication (Dexamethasone; Bausch\&Lomb).

\section{RESULTS}

\section{Subject Accountability, Demographics, and Preoperative Parameters}

This prospective consecutive cohort included 125 total eyes (81 Combined, 44 Standalone) of a single glaucoma surgeon at an academic hospital in Germany. Nearly all eyes $(121 / 125$ or 97\%) completed 5 years of follow-up. Preoperative parameters are shown in Table 1 . These were patients carrying a relatively substantial preoperative treatment burden, with overall mean preoperative IOP of $23.5 \pm 6.2 \mathrm{mmHg}$ on $2.68 \pm 1.02$ mean medications. All but 1 eye (>99\%) were on medication(s) preoperatively; $38 \%$ of eyes had undergone prior glaucoma surgery. In comparison to Combined eyes, Standalone eyes had slightly higher mean IOP and medications, and higher proportions of eyes with history of prior glaucoma surgery (50\% vs. $32 \%$, respectively) and eyes on $\geq 3$ glaucoma medications $(75 \%$ vs. $54 \%$, respectively).

\section{Intraocular Pressure}

In the Overall cohort $(n=125)$, mean IOP decreased to $14.1 \pm 1.8 \mathrm{mmHg}$ at 5 years $(40 \%$ reduction vs. preoperative; $p<0.001$ ) (Fig. 1a). $A \geq 20 \%$ IOP reduction versus preoperative was achieved in $83 \%$ of eyes, with all but 1 eye (>99\%) maintaining or reducing IOP versus preoperative (the one remaining eye increased from 15 to $16 \mathrm{mmHg}$ ). The proportion of eyes with $\mathrm{IOP} \leq 18 \mathrm{mmHg}$ rose to $98 \%$ (vs. $21 \%$ preoperatively; $p<0.001)$; and eyes with IOP $\leq 15 \mathrm{mmHg}$ rose to $81 \%$ (vs. $<2 \%$ preoperatively; $p<0.001$ ) (Fig. $2 \mathrm{a}$ ).

The IOP outcomes in the Combined and Standalone subgroups were similar to those of the Overall cohort. In Combined eyes, mean IOP decreased from $22.6 \mathrm{mmHg}$ preoperatively to $13.8 \mathrm{mmHg}$ at 5 years $(39 \%$ reduction; $p<0.001$ ) (Fig. 1b). All Combined eyes (100\%) achieved IOP $\leq 18 \mathrm{mmHg}$ (vs. $27 \%$ preoperatively; $p<0.001$ ), and $86 \%$ of eyes had $\mathrm{IOP} \leq 15 \mathrm{mmHg} \quad$ (vs. $1 \%$ preoperatively; $p<0.001$ ) (Fig. 2b).

In Standalone eyes, mean IOP reduced from $25.3 \mathrm{mmHg}$ to $14.6 \mathrm{mmHg}$ ( $42 \%$ reduction, $p<0.001$ ) (Fig. 1c). There was a significant rise in the proportion of eyes with IOP $\leq 18 \mathrm{mmHg}$ $(95 \%$ at 5 years vs. $9 \%$ preoperatively, $p<0.001)$, and with IOP $\leq 15 \mathrm{mmHg}(71 \%$ at 5 years vs. $2 \%$ preoperatively, $p<0.001$ ) (Fig. 2c).

\section{Medication Use}

In the Overall cohort, mean number of medications at 5 years decreased to $0.77 \pm 0.82$ medications (71\% reduction vs. preoperative; $p<0.001$ ) (Fig. 3a). At 5 years, $46 \%$ of eyes were medication-free (vs. 1 eye or $<1 \%$ preoperatively; $p<0.001$ ), while $<2 \%$ of eyes were using $\geq 3$ medications (versus $62 \%$ preoperatively; $p<0.001)$. All eyes $(100 \%)$ were able to maintain or reduce their medication regimen versus preoperative (Fig. 4a).

Within the Combined and Standalone subgroups, the 5-year medication reductions were similar to those of the Overall cohort. In Combined eyes, mean number of medications reduced from 2.52 to 0.78 medications $(69 \%$ reduction; $p<0.001$ ) (Fig. 3b). The proportion of medication-free eyes rose to $44 \%$ (vs. 1 eye or $1 \%$ preoperatively; $p<0.001$ ); inversely, only 1 


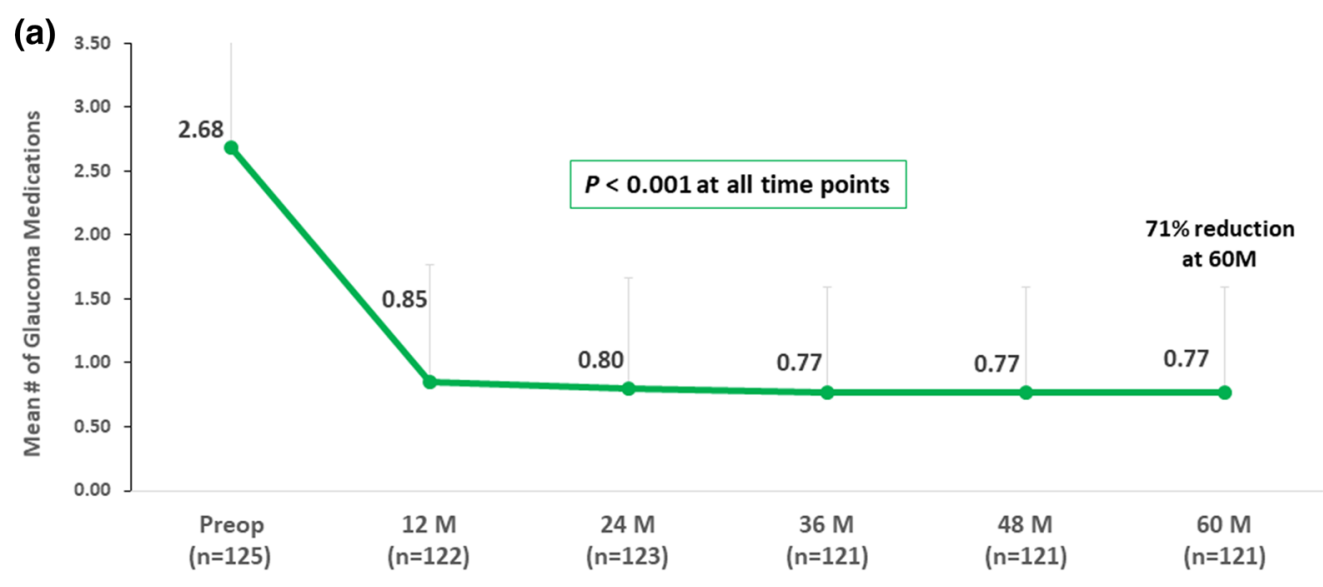

$M=$ months; Preop = preoperative Vertical bars represent standard deviation

(b)

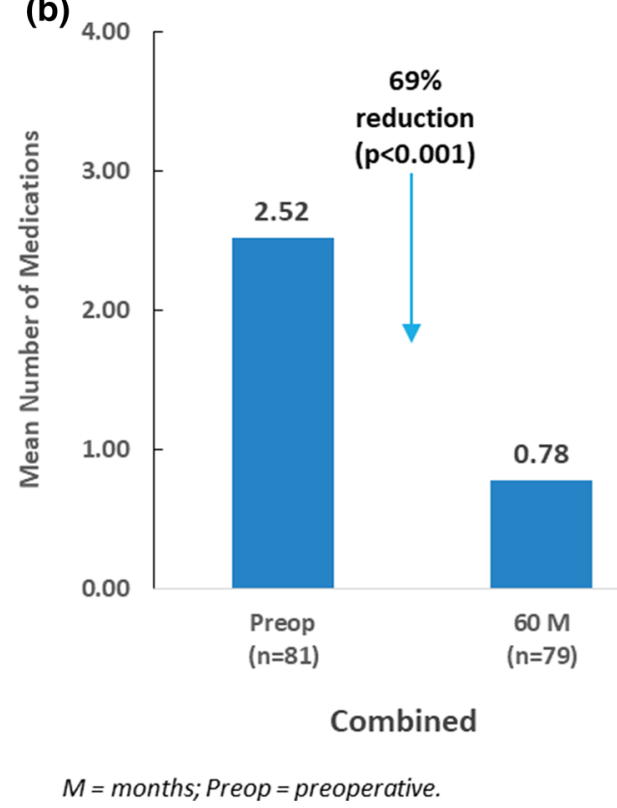

(c)

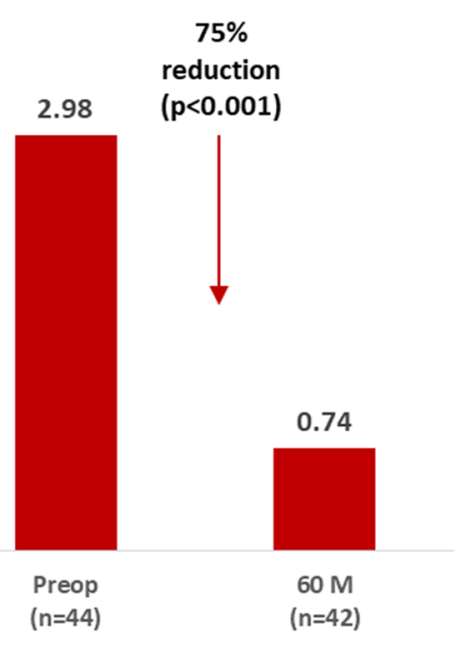

Standalone

Fig. 3 a Mean number of medications through 5 years postoperative in overall cohort. b (left) Mean Medication burden at 5 years versus preoperative: combined subgroup. $\mathbf{c}$ (right) Mean IOP at 5 years versus preoperative: standalone subgroup

eye (1\%) at 5 years was on $\geq 3$ medications (vs. $54 \%$ of eyes preoperatively; $p<0.001$ ) (Fig. $4 \mathrm{~b}$ ). All Combined eyes maintained or reduced medication burden versus preoperative.

In Standalone eyes, mean number of medications reduced from 2.98 preoperatively to 0.74 medications at 5 years $(75 \%$ reduction; $p<0.001$ ) (Fig. 3c). The proportion of medication-free eyes rose to $50 \%$ (vs. 0 eyes preoperatively; $p<0.001)$; inversely, only 1 eye $(2 \%)$ at
5 years was on $\geq 3$ medications (vs. $75 \%$ of eyes preoperatively; $p<0.001$ ) (Fig. 4c). All Standalone eyes achieved the same or lower medication burden versus preoperative.

\section{Safety Assessment}

All patients underwent successful implantation of 2 iStent inject stents either as a sole procedure 
(a)

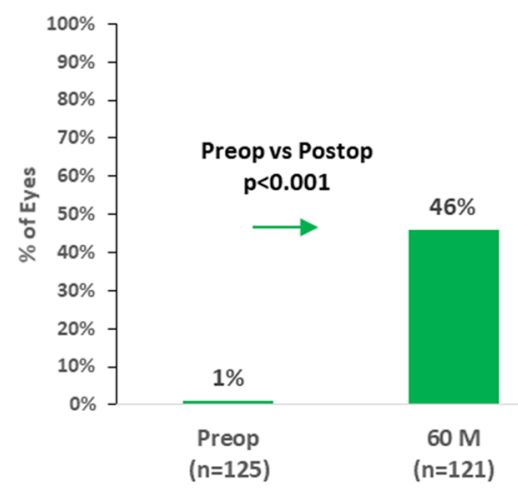

Medication-Free

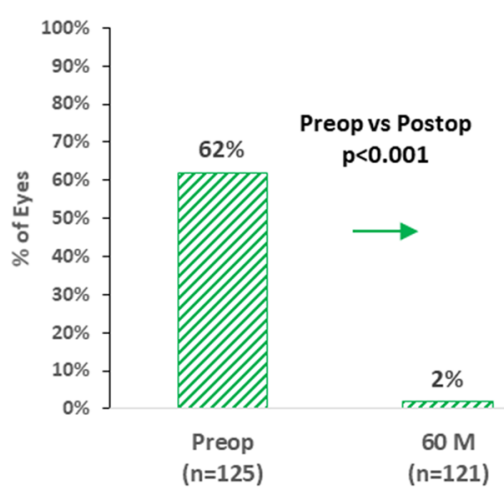

3-5 Medications

$100 \%$ of eyes had the same or lower medication burden vs preop

$M=$ months; Preop = preoperative;

$P$ Postop $=$ postoperative $1 O P=$ intraocular pressure.

(b)

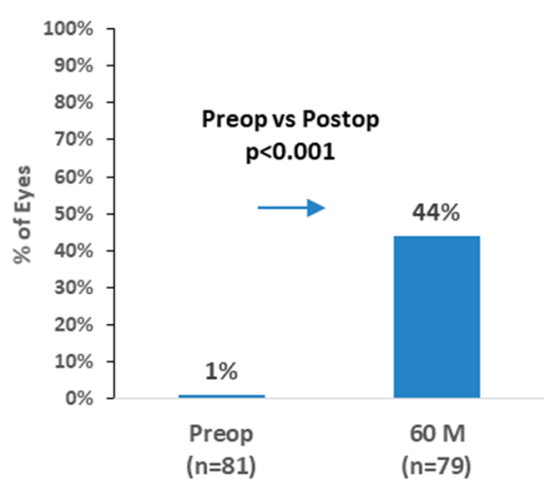

Medication-Free

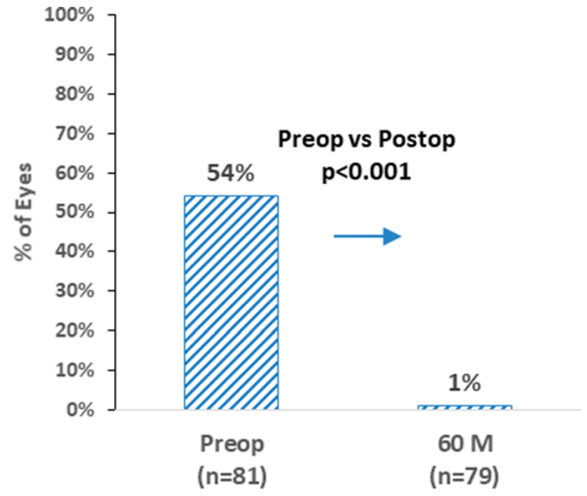

3-5 Medications

$M=$ months; Preop = preoperative;

Postop $=$ postoperative $I O P=$ intraocular pressure.

(c)

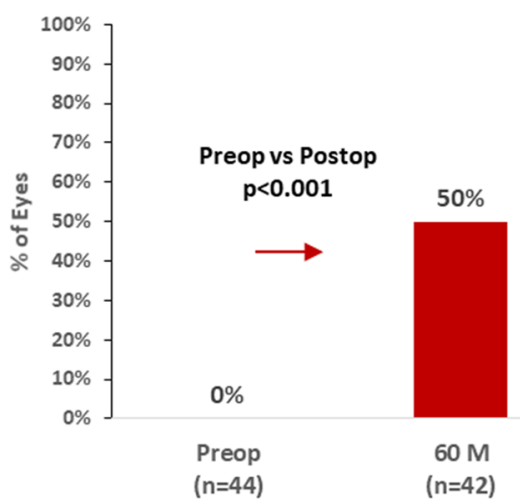

Medication-Free

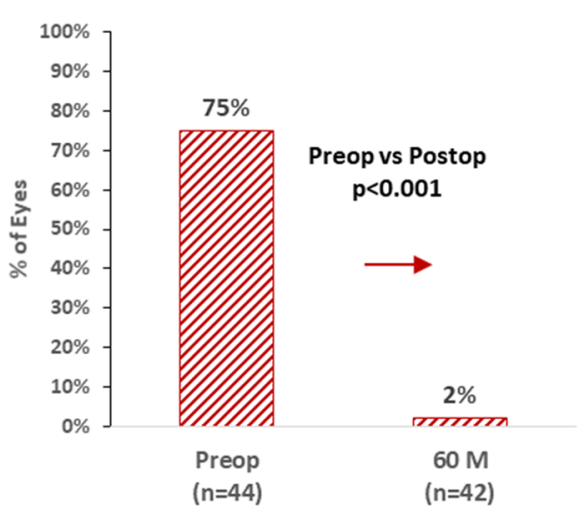

3-5 Medications

$M=$ months; Preop = preoperative;

Posto $p=$ postoperative $I O P=$ intraocular pressure. 
4Fig. 4 a Proportional analysis of medication outcomes at 5 years versus preoperative in overall cohort: eyes medication-free, eyes on 3-5 medications. b Proportional analysis of medication outcomes at 5 years versus preoperative in combined subgroup: eyes medication-free, eyes on 3-5 medications. c Proportional analysis of medication outcomes at 5 years versus preoperative in standalone subgroup: eyes medication-free, eyes on 3-5 medications

or following standard phacoemulsification cataract surgery. One intraoperative event occurred: a Standalone case with mild intraoperative hyphema which resolved without intervention or sequelae by Week 1 . Postoperative adverse events during the first 3 years have been described in prior publications; these events were generally mild, transient, and self-resolved or were treated conservatively without sequelae $[3,4]$. They included 2 Combined eyes with mild corneal edema on Day 1 which resolved by Day 5 postoperatively; 2 eyes of one Standalone patient with cataract progression at Month 3, and 1 Standalone eye with uveitis at Month 24, which resolved within 3 weeks with topical anti-inflammatory medication. During 2 additional years of follow-up, no new adverse events occurred. Throughout follow-up there were no stent-related complications; no signs of chronic inflammation such as peripheral anterior synechiae (PAS); and no cases of hypotony, endophthalmitis, corneal compromise, stent obstruction, myopic shift, or choroidal detachment.

Over the course of 5-year follow-up, a total of 5 eyes (3 Combined, 2 Standalone) underwent an additional glaucoma procedure due to the need for additional IOP-lowering beyond what had been achieved with stent implantation. The procedures, all of which occurred at Month 1 or Month 6 postoperative, included cyclophotocoagulation (CPC) in 3 eyes and XEN implantation in 2 eyes. No filtering surgeries were performed in any eye throughout the 5 years.

\section{Long-term Disease Stability}

Long-term indicators of disease stability were measured and compared between the preoperative and 5-year visits. No significant differences were observed in visual fields, retinal nerve fiber layer thickness, or C:D ratio in either the Combined or Standalone subgroups over the course of the study. Regarding VF stability over time, average VF MD in the Combined subgroup was $-6.6 \pm 2.8 \mathrm{db}$ preoperatively versus $-6.7 \pm 2.6 \mathrm{db}$ at 5 years $(p=0.462)$. In the Standalone subgroup, average VF MD was $-7.0 \pm 3.5 \mathrm{db}$ preoperatively versus $-7.1 \pm 3.3$ $\mathrm{db}$ at 5 years $(p=0.502)$. Regarding RNFL stability over time, mean RNFL thickness in the Combined subgroup was $81.4 \pm 12.3 \mu \mathrm{m}$ preoperatively versus $80.2 \pm 13.5 \mu \mathrm{m}$ at 5 years $(p=0.497)$. In the Standalone subgroup, mean RNFL thickness was $82.1 \pm 13.6 \mu \mathrm{m}$ preoperatively versus $80.9 \pm 14.7 \mu \mathrm{m}$ at 5 years $(\mathrm{p}=0.194)$. Regarding C:D stability over time, mean C:D ratio in the Combined subgroup was $0.74 \pm 0.17$ preoperatively versus $0.76 \pm 0.14$ at 5 years $(p=0.324)$. In the Standalone subgroup, mean C:D ratio was $0.78 \pm 0.18$ preoperatively versus $0.79 \pm 0.16$ at 5 years $(p=0.386)$.

\section{DISCUSSION}

This prospective consecutive longitudinal cohort, with $97 \%$ of the 125 eyes followed through 5 years, constitutes one of the largest and longest-term datasets to date on iStent inject. Given that Germany was one of the first countries to adopt this technology, the present dataset provides a valuable window on iStent inject outcomes over the long term. These outcomes included stable and significant reductions in IOP and medications, together with excellent safety and $100 \%$ of eyes avoiding filtration surgery. Although the cohort had relatively high glaucoma medication burden preoperatively, nearly half of eyes were medication-free by the end of 5 years, while mean IOP decreased by nearly $10 \mathrm{mmHg}$. The observed reductions in IOP and medication were similar in magnitude and consistency over time regardless of whether stenting was performed as a standalone surgery or in combination with phacoemulsification.

A number of noteworthy attributes may be highlighted in this study. First, it provides some 
of the first 5-year data on iStent inject in a realworld clinical population, as noted above. Patient accountability was nearly $100 \%$ over the entirety of follow-up, supporting the credibility and strength of the findings. The cohort included Combined and Standalone subgroups within the hands of a single surgeon at a single site and drawn from the same patient base. Such uniformity helps reduce confounding when comparing across subgroups and when distinguishing the IOP-lowering effect of the stents versus that of phacoemulsification.

The percent reductions in IOP and medications were similar for both Combined and Standalone subgroups, even though Standalone eyes had higher preoperative IOP, medication burden, and history of prior glaucoma surgery. This is a strong confirmation that the stentsrather than the cataract extraction-are producing the change. The independent effect of the stents is also validated by the consistency of mean IOP and medications throughout 5 years of follow-up (in contrast to the typically temporary ocular hypotensive effect of phacoemulsification). In Standalone eyes, as in combined eyes, the IOP and medication reductions at 5 years were similar to those observed at 3 years, suggesting no diminution of treatment effect over time. Such stability is noteworthy, given the natural history of glaucoma as an otherwise progressive, steadily advancing disease.

In comparison to the majority of existing literature, the postoperative medication burden (both mean and percent reduction vs. baseline) was comparable to what has been reported previously in combined and standalone iStent inject cohorts [7-32]. The postoperative mean IOP was also in the range of prior iStent inject reports, although the current study's pre-versuspostoperative percent reduction was greater than in many other studies. Given the overall similarity and consistency of postoperative IOP ranges across the reports, the greater postoperative percent reduction in the present study is possibly a function of higher starting pressure, which is known to allow for greater percent IOP reductions postoperatively [8-12].

Among the publications on iStent inject, two in particular may be informative against which to compare our study. For the Combined subgroup, we can consider our findings in the context of the largest multicenter real-world study to-date on combined iStent inject implantation [15]. That study, by Clement et al., included 340 eyes from 9 surgeons across Australia. They reported final IOP of $13.7 \mathrm{mmHg}$ at 2 years postoperative (a 16\% reduction vs. a relatively low baseline IOP of $16.4 \mathrm{mmHg}$ ), with $77 \%$ of eyes achieving IOP $\leq 15 \mathrm{mmHg}$ and mean medication burden reducing by $67 \%$ (from 1.49 to 0.49 mean medications) [15]. In comparison, the Combined subgroup in our cohort experienced a final postoperative IOP of $13.8 \mathrm{mmHg}$ (a 39\% reduction vs. a baseline IOP of $22.6 \mathrm{mmHg}$ ), with $86 \%$ of eyes achieving IOP $\leq 15 \mathrm{mmHg}$ and mean medication burden reducing by $69 \%$ (from 2.52 to 0.78 mean medications). For the Standalone subgroup in our cohort, the IOP outcomes are closely aligned with the largest and most comprehensive systematic review and meta-analysis to-date of standalone iStent and iStent inject implantation [7]. That publication, by Healey et al., analyzed 13 different studies and reported that they all centered around a final mean IOP of $13.0-16.5 \mathrm{mmHg}$ through up to 5 years postoperative, starting from a mean preoperative IOP of $19.1-25.3 \mathrm{mmHg}$. Similarly, the Standalone subgroup in our cohort experienced a final IOP of $14.6 \mathrm{mmHg}$ at 5 years postoperative, starting from a mean preoperative IOP of $25.3 \mathrm{mmHg}$.

As is characteristic of the iStent family of devices, safety outcomes in this study were highly favorable. Notably, there were no intraoperative complications and no filtration surgery in any eye over 5 years of follow-up. Given their substantial preoperative disease burden (mean IOP $23.5 \mathrm{mmHg}$ on 2.68 medications) and history of prior glaucoma surgery (38\% of eyes), many of these patients would possibly have needed higher-risk filtration surgery at some point during the 5 years of follow-up if they had not stabilized their IOP with iStent inject implantation.

The study was limited by its unmasked, single-arm, non-randomized design. Patients comprised a consecutive sample of all cases of a single surgeon; this increases generalizability 
but also makes it more difficult to account for non-quantifiable differences in preoperative characteristics. Patients did not complete medication washouts, as this was not indicated nor appropriate in the patients' regular clinical care. All patients were non-Hispanic white and of German nationality, so the reader must exercise discernment when extrapolating outcomes to other populations. Within the Combined subgroup, the IOP-lowering effect of cataract extraction could not be separated from that of the concurrent stent implantation. However, post-phacoemulsification IOP reduction in medicated glaucoma patients is known to be modest (less than $2 \mathrm{mmHg}$ on average) [33-35], and IOP typically starts returning to baseline levels after the first year postoperative [35-38]. Therefore, our study's findings of consistent IOP reduction through 5 years, and similar reductions in Combined and Standalone patients, both corroborate that phacoemulsification is no longer exerting an effect on IOP outcomes. Future areas for investigation could include expanded analyses of specific glaucoma subtypes (such as PXG), stratification by preoperative glaucoma severity or surgical history, and inclusion of the newest iStent inject model, the wide-flanged iStent inject W (released in Germany in 2020).

\section{CONCLUSIONS}

This study contributes valuable 5-year data on iStent inject implantation either in combination with cataract surgery or as a sole procedure. The same cohort was prospectively identified and followed longitudinally, with nearly all patients reaching their 5-year visit. Although patients had a relatively high glaucoma treatment burden preoperatively, nearly half of eyes were medication-free by 5 years, while mean IOP reduced by nearly $10 \mathrm{mmHg}$. The degree of IOP and medication reduction was similar in both Combined and Standalone subgroups, validating the stents' independent effect apart from that of cataract extraction. The treatment effect was also consistent visit-to-visit throughout follow-up, confirming the sustained 5-year effectiveness of the stents. Disease stability was also confirmed, with no significant change in VF, RNFL, or C:D ratio at 5 years compared to preoperative. And, finally, since outcomes were recorded in a real-world patient population as part of routine clinical care, they can be applicable to surgeons making treatment decisions with their patients in combined and standalone settings.

\section{ACKNOWLEDGEMENTS}

Funding. Open Access funding enabled and organized by Projekt DEAL. An unrestricted research grant and editorial assistance were provided by Glaukos Corporation. Glaukos Corporation also funded the Journal's Rapid Service and Open Access Fees. All authors had full access to all of the data in this study and take complete responsibility for the integrity of the data and accuracy of the data analysis.

Authorship. All authors meet the International Committee of Medical Journal Editors (ICMJE) criteria for authorship for this article, take responsibility for the integrity of the work as a whole, and have given their approval for this version to be published. Editorial assistance in the preparation of this article was provided by Dr. Dana M. Hornbeak, MD, MPH of Glaukos Corporation.

Author Contributions. Fritz H. Hengerer: concept and design, completion of surgeries, preoperative/postoperative management, data collection, statistical analysis, manuscript drafting. Gerd U. Auffarth: concept and design, preoperative/postoperative management, data collection, manuscript drafting. Ina ConradHengerer: concept and design, preoperative/postoperative management, statistical analysis, manuscript drafting.

Disclosures. Fritz H. Hengerer: travel support, research grants, and lecture fees from Glaukos Corporation. Gerd U. Auffarth: travel support, research grants, and lecture fees from Glaukos Corporation. Ina Conrad-Hengerer: 
research grants, and lecture fees from Glaukos Corporation.

Compliance with Ethics Guidelines. All procedures performed were in accordance with the ethical standards of the institutional and/or national research committee (the Institutional Review Board (IRB) of the University of Heidelberg), and with the 1964 Helsinki declaration and its later amendments or comparable ethical standards. Informed consent was obtained from all individual participants included in the study.

Data Availability. The datasets generated during and/or analyzed during the current study are available from the corresponding author on reasonable request.

Open Access. This article is licensed under a Creative Commons Attribution-NonCommercial 4.0 International License, which permits any non-commercial use, sharing, adaptation, distribution and reproduction in any medium or format, as long as you give appropriate credit to the original author(s) and the source, provide a link to the Creative Commons licence, and indicate if changes were made. The images or other third party material in this article are included in the article's Creative Commons licence, unless indicated otherwise in a credit line to the material. If material is not included in the article's Creative Commons licence and your intended use is not permitted by statutory regulation or exceeds the permitted use, you will need to obtain permission directly from the copyright holder. To view a copy of this licence, visit http:// creativecommons.org/licenses/by-nc/4.0/.

\section{REFERENCES}

1. Boland MV, Corcoran KJ, Lee AY. Changes in Performance of Glaucoma Surgeries 1994 through 2017 Based on Claims and Payment Data for United States Medicare Beneficiaries. Ophthalmol Glaucoma. 2021;S2589-4196(21):00032-6. https://doi. org/10.1016/j.ogla.2021.01.004.
2. Businesswire.com Press Release August 23, 2021. "Glaukos Announces Market-Leading Clinical Milestone of 200 Peer-Reviewed Publications on iStent Technologies." https://www.businesswire. com/news/home/20210823005049/en/GlaukosAnnounces-Market-Leading-Clinical-Milestone-of200-Peer-Reviewed-Publications-on-iStent $\%$ C2\% AE-Technologies.

3. Hengerer FH, Auffarth GU, Riffel C, Conrad-Hengerer I. Prospective, non-randomized, 36-month study of second-generation trabecular micro-bypass stents with phacoemulsification in various types of glaucoma. Ophthalmol Ther. 2018;7(2):405-15.

4. Hengerer FH, Auffarth GU, Riffel C, Conrad-Hengerer I. Second-generation trabecular micro-bypass stents as standalone treatment for glaucoma: a 36-month prospective study. Adv Ther. 2019;36(7): 1606-17.

5. Samuelson TW, Sarkisian SR Jr, Lubeck DM, Stiles MC, Duh Y-J, Romo EA, Giamporcaro JE, Hornbeak DM, Katz LJ. for the iStent inject study group, prospective, randomized, controlled pivotal trial of iStent inject trabecular micro-bypass in primary open-angle glaucoma and cataract: two-year results. Ophthalmology. 2019;126(6):811-21.

6. Huang AS, Penteado RC, Papoyan V, Voskanyan L, Weinreb RN. Aqueous angiographic outflow improvement after trabecular micro-bypass in glaucoma patients. Ophthalmol Glaucoma. 2019;2: 11-21.

7. Healey PR, Clement CI, Kerr NM, Tilden D, Aghajanian L. Standalone iStent trabecular micro-bypass glaucoma surgery: a systemic review and metaanalysis. J Glaucoma. 2021. https://doi.org/10. 1097/IJG.0000000000001805.

8. Ferguson TJ, Dockter Z, Bleeker A, Karpuk KK, Schweitzer J, Ibach MJ, Berdahl JP. iStent inject trabecular microbypass stent implantation with cataract extraction in open-angle glaucoma: early clinical experience. Eye Vis (Lond). 2020;20(7):28.

9. Ferguson TJ, Mechels KB, Dockter Z, et al. iStent trabecular microbypass stent implantation with phacoemulsification in patients with open-angle glaucoma: 6-year outcomes. Clin Ophthalmol. 2020;14:1859-66. https://doi.org/10.2147/OPTH. S247910.

10. Ferguson T, Swan R, Ibach M, Schweitzer J, Sudhagoni R, Berdahl JP. Evaluation of a trabecular microbypass stent with cataract extraction in severe primary open-angle glaucoma. J Glaucoma. 2018;27(1):71-6.

11. Ferguson TJ, Ibach M, Schweitzer J, Karpuk K, Stephens JD, Sudhagoni R, Berdahl JP. Trabecular 
microbypass stent implantation in pseudophakic eyes with open-angle glaucoma: Long-term results. J Cataract Refract Surg. 2019;45(4):414-20.

12. Salimi A, Watt H, Harasymowycz P. Three-year outcomes of second-generation trabecular microbypass stents (iStent inject) with phacoemulsification in various glaucoma subtypes and severities. J Glaucoma. 2021;30(3):266-75.

13. Ferguson TJ, Swan RJ, Bleeker A, et al. Trabecular microbypass stent implantation in pseudoexfoliative glaucoma: long-term results. J Cataract Refract Surg. 2020;46(9):1284-9.

14. Ferguson TJ, Ibach M, Schweitzer J, Karpuk KL, Stephens JD, Berdahl JP. Trabecular microbypass stent implantation with cataract extraction in pigmentary glaucoma. Clin Exp Ophthalmol. 2020;48(1):37-43.

15. Clement C, Howes F, Ioannidis AS, Shiu M, Manning D, Lusthaus J, Lim R, Skalicky SE, Goodwin T. Two-year multicenter outcomes of iStent inject trabecular micro-bypass stents combined with phacoemulsification in various types of glaucoma and ocular hypertension. Clin Ophthalmol. 2020;14:3507-17.

16. Guedes RAP, Gravina DM, Paletta Guedes VM, Chaoubah A. Two-year comparative outcomes of first- and second-generation trabecular micro-bypass stents with cataract surgery. Clin Ophthalmol. 2021;5(15):1861-73.

17. Neuhann R, Neuhann T. Second-generation trabecular micro-bypass stent implantation: retrospective analysis after 12- and 24-month follow-up. Eye Vis (Lond). 2020;10(7):1.

18. Salimi A, Abu-Nada M, Harasymowycz P. Matched cohort study of Cataract surgery with and without trabecular micro-bypass stent implantation in primary angle-closure glaucoma. Am J Ophthalmol. 2021. https://doi.org/10.1016/j.ajo.2020.12.032.

19. Salimi A, Clement C, Shiu M, Harasymowycz P. Second-generation trabecular micro-bypass (iStent inject) with cataract surgery in eyes with normaltension glaucoma: one-year outcomes of a multicentre study. Ophthalmol Ther. 2020;9(3):585-96.

20. Berdahl J, Voskanyan L, Myers JS, Katz LJ, Samuelson TW. iStent inject trabecular micro-bypass stents with topical prostaglandin as standalone treatment for open-angle glaucoma: 4-year outcomes. Clin Exp Ophthalmol. 2020;48(6):767-74.

21. Lindstrom R, Sarkisian SR, Lewis R, Hovanesian J, Voskanyan L. Four-year outcomes of two secondgeneration trabecular micro-bypass stents in patients with open-angle glaucoma on one medication. Clin Ophthalmol. 2020;14:71-80.

22. Samuelson TW, Singh IP, Williamson BK, Falvey H, Lee WC, Odom D, McSorley D, Katz LJ. Quality of life in primary open-angle glaucoma and cataract: an analysis of VFQ-25 and OSDI from the iStent inject ${ }^{\circledR}$ Pivotal Trial. Am J Ophthalmol. 2021. https://doi.org/10.1016/j.ajo.2021.03.007.

23. Schweitzer JA, Hauser WH, Ibach $\mathrm{M}$, et al. Prospective interventional cohort study of ocular surface disease changes in eyes after trabecular micro-bypass Stent(s) implantation (iStent or iStent inject) with phacoemulsification. Ophthalmol Ther. 2020;9(4):941-53.

24. Al Habash A, Nagshbandi AA. Quality of life after combined cataract and minimally invasive glaucoma surgery in glaucoma patients. Clin Ophthalmol. 2020;14:3049-56.

25. Jabłońska J, Lewczuk K, Konopińska J, Mariak Z, Rękas M. Microinvasive glaucoma surgery: a review and classification of implant-dependent procedures and techniques. Acta Ophthalmol. 2021. https:// doi.org/10.1111/aos.14906.

26. Ang BCH, Tecson ICRO, Hu JYW, Kan JTC, Yip LWL. 12-month outcomes of combined phacoemulsification and iStent inject in Asian eyes with normal tension glaucoma: a single-centre experience. Int Ophthalmol. 2021. https://doi.org/ 10.1007/s10792-021-02033-3.

27. Silveira Seixas RC, Balbino M, Basile Neto A, de Alcantara Almeida Costa A, Jordão MLS, Russ HHA. Mid-term evaluation of iStent Inject ${ }^{\circledR}$ trabecular micro-bypass stent implantation with or without phacoemulsification: a retrospective study. Clin Ophthalmol. 2020;14:4403-13.

28. Al Habash A, Otaif W. Surgical outcomes of combined 2nd-generation trabecular microbypass (iStent Inject) and cataract surgery for the treatment of primary open-angle glaucoma in the Saudi population. Ophthalmol Ther. 2021. https://doi.org/ 10.1007/s40123-021-00380-z.

29. Ioannidis AS, Toteberg-Harms M, Hamann T, Hodge C. Refractive outcomes after trabecular micro-bypass stents (iStent Inject) with cataract extraction in open-angle glaucoma. Clin Ophthalmol. 2020;14:517-24.

30. Manning D. Real-world case series of iStent or iStent Inject trabecular micro-bypass stents combined with cataract surgery. Ophthalmol Ther. 2019;8(4): 549-61.

31. Singh IP, Sarkisian S, Hornbeak D, Katz LJ, Samuelson T, iStent inject Study Group. Treatment 
success across different levels of preoperative disease burden: stratified two-year outcomes from the pivotal trial of iStent inject ${ }^{\circledR}$ trabecular micro-bypass in primary open-angle glaucoma and cataract. Clin Ophthalmol. 2021;15:3231-40.

32. Voskanyan L, Garcia-Feijoo J, Belda J, Fea A, Jünemann A, Baudouin C, Synergy Study Group. Prospective, unmasked evaluation of the iStent inject system for open-angle glaucoma: synergy trial. Adv Ther. 2014;31(2):189-201.

33. Vizzeri G, Weinreb RN. Cataract surgery and glaucoma. Curr Opin Ophthalmol. 2010;21(1):20-4.

34. Friedman DS, Jampel HD, Lubomski LH, et al. Surgical strategies for coexisting glaucoma and cataract: an evidence-based update. Ophthalmology. 2002;109:1902-13.

35. Poley BJ, Lindstrom RL, Samuelson TW. Long-term effects of phacoemulsification with intraocular lens implantation in normotensive and ocular hypertensive eyes. J Cataract Refract Surg. 2008;34(5): 735-42.

36. Shingleton BJ, Gamell LS, O'Donoghue MW, Baylus SL, King R. Long-term changes in intraocular pressure after clear corneal phacoemulsification: normal patients versus glaucoma suspect and glaucoma patients. J Cataract Refract Surg. 1999;25(7):885-90.

37. Mathalone N, Hyams M, Neiman S, et al. Long-term intraocular pressure control after clear corneal phacoemulsification in glaucoma patients. J Cataract Refract Surg. 2005;31:479-83.

38. Kim DD, Doyle JW, Smith MF. Intraocular pressure reduction following phacoemulsification cataract extraction with posterior chamber lens implantation in glaucoma patients. Ophthalmic Surg Lasers. 1999;30(1):37-40. 